\title{
Research Paper \\ Mediating Role of Resilience and Hope in the Relationship Between Perceived Social Support and Life Satisfaction in Orphaned Adolescents
}

\author{
Abbas Ali Tatar', *Abbas Nesayan² ${ }^{2}$, Roghayeh Asadi Gandomani $^{2}$
}

1. MA., Department of Psychology, Faculty of Humanities, Birjand Branch, Islamic Azad University, Birjand, Iran.

2. Assistant Professor, Department of Psychology, Faculty of Humanities, University of Bojnord, Bojnord, Iran.

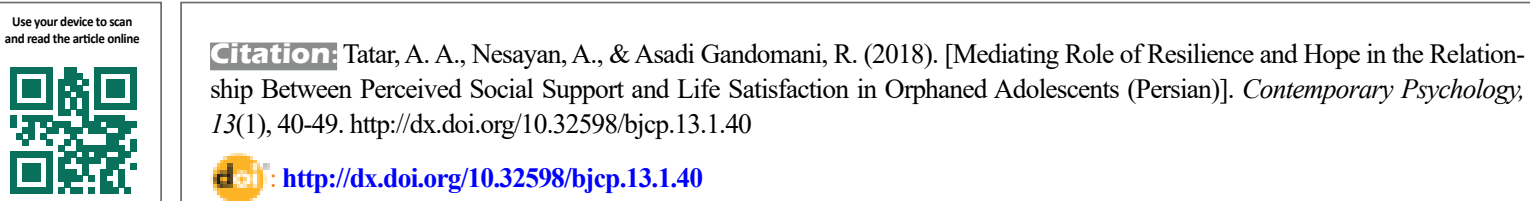

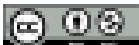

Received: 23 Jul 2017

Accepted: 05 Dec 2017

Available Online: 21 Mar 2018

\section{Key words:}

Resilience, Hope, Perceived social support Life satisfaction

\section{A B STRACT}

Objectives Life satisfaction is an important psychological concept that is associated with person's general assessment of life. Many variables can affect life satisfaction. This research was conducted to determine the relationship between perceived social support and life satisfaction with mediating role of resilience and hope.

Methods A total of 270 adolescents who had records at Imam Khomeini Relief Foundation, Social Security Organization and Social Welfare Organization in Zabol City, Iran participated in this study. Participants completed the questionnaires of satisfaction with life, multidimensional scale of perceived social support, Connor-Davidson resilience scale and trait hopes scale.

Results The results of this study showed that the relationship between perceived social support and life satisfaction with mediating of hope and resilience fit with the data. The obtained data also revealed a positive, direct and significant relationship between perceived social support with hope, resilience and life satisfaction. In addition, hope and resilience had a positive, direct, and significant effect on life satisfaction.

Conclusion Based on the study results, we should pay attention to predictive variables such as social support and intermediary mechanisms such as resilience and hope for the development and improvement of life satisfaction.

* Corresponding Author:

Abbas Nesayan, PhD.

Address: Department of Psychology, Faculty of Humanities, University of Bojnord, Bojnord, Iran.

Tel: +98 (912) 6872706

E-mail: anesayan@gamil.com 


\title{
نقش واسطداى تابآورى و اميدوارى در رابطه بين حمايت اجتماعى اداركشده و رضايت از زندكى نوجوانان بىسريرست تابت
}

\author{
عباسعلى تاتار'،" عباس نسائيان’ •، رقيه اسدى كندمانى'
}

ا- كارشناس ارشد، كروه روانشناسى، دانشكده علوم انسائي، واحد بير جند، دانشكاه آزاد اسلامى، بير جنده ايران.

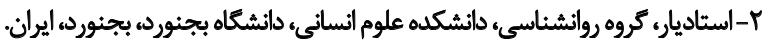

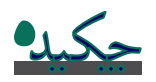

هدف رضايت ازز زندكى يك سازه مهمه روانشئاختى و به معنى ارزيابى كلى فرد از زئدكى است. متغيرهاى بسيارى مىتوانند در ارتباط با رضايت

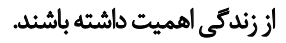

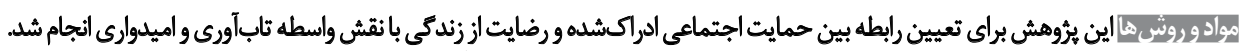

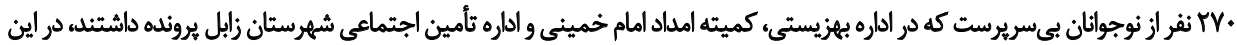

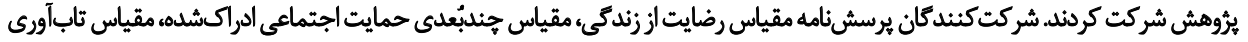
و مقياس اميدواري صفتى را تكميل كردند

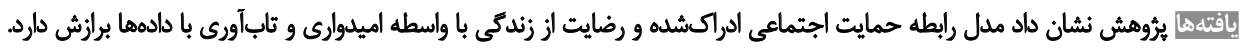

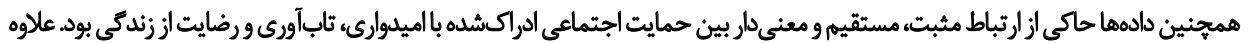

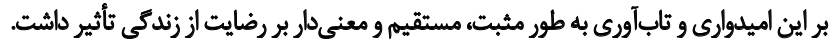

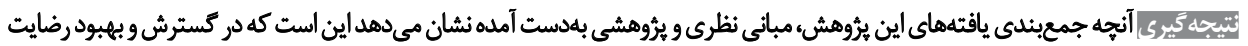

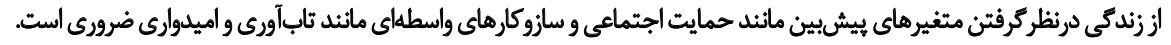

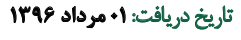

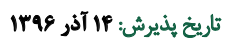

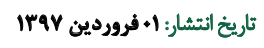

با آنجه او به عنوان يك استاندارد مطلوب در نظر دارد، تعريف

dales

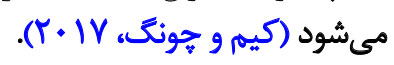

دايثر' و همكاران (1999) دو رويكرد را مشخص كردهاند كه ميتواند رضايث از زندگى افراد را تحث ثأثير قرار دهد، اين

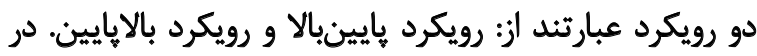

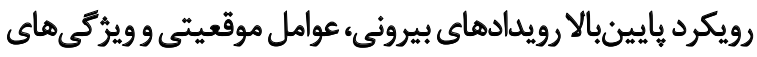
جمعيتشناختى و در رويكرد بالإيايين فرايندهاي دروني موردين مورديني توجه قرار كرفتهاند.

انسانها موجوداتى بسيار اجتماعى هستند كه زندكى آنها

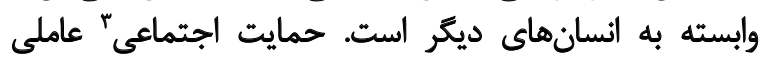

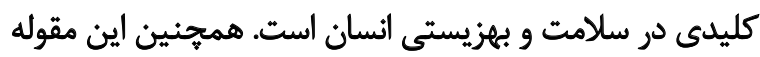

2.Diener

3. Social support

1. Life satisfaction

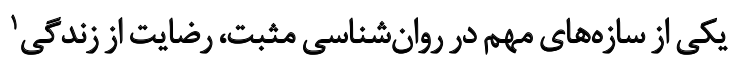

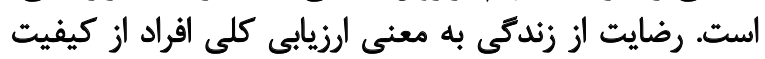

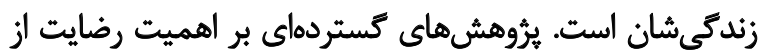

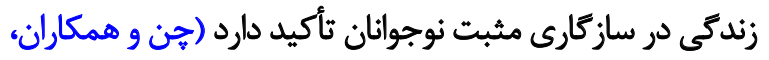

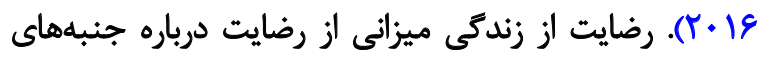

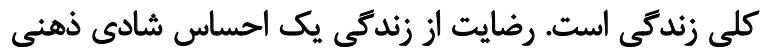

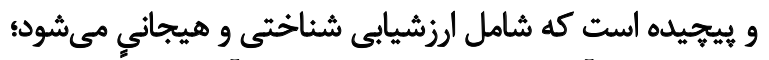

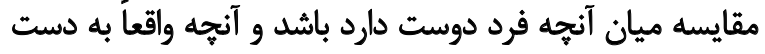

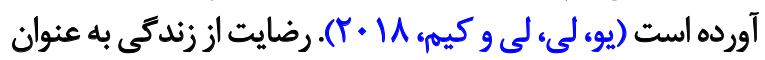

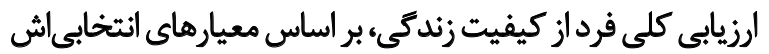

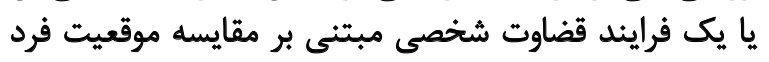

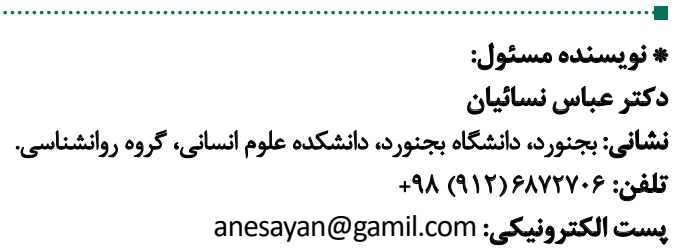

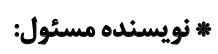

دكتر عباس نسائيان

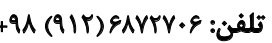
يست الكترونيكي: anesayan@gamil.com 


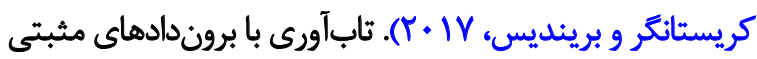

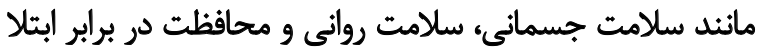

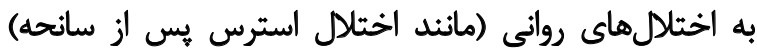

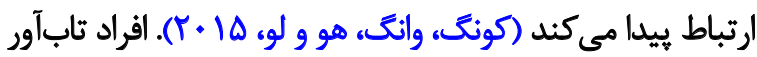

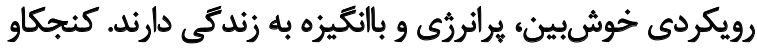

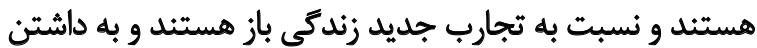

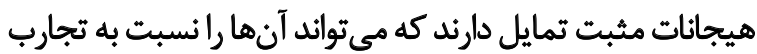

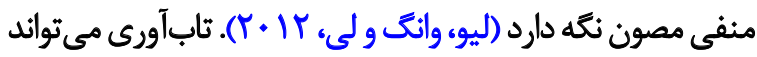

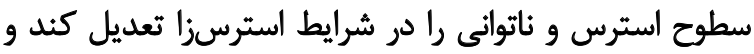

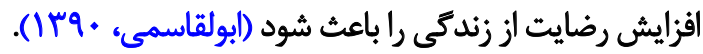

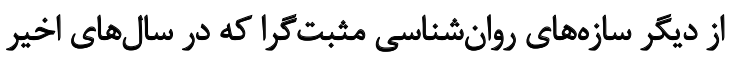

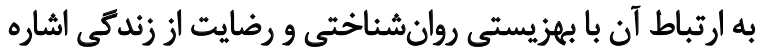

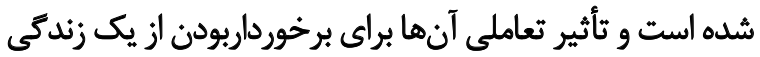

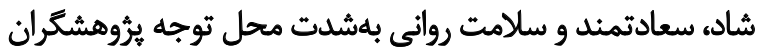

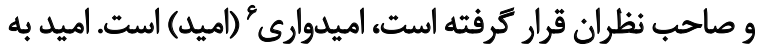

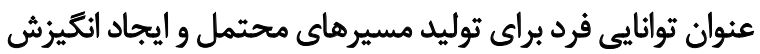

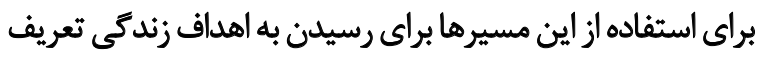

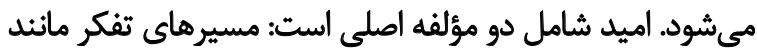

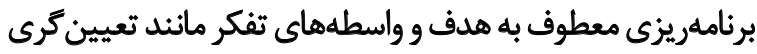

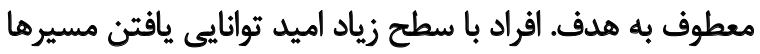

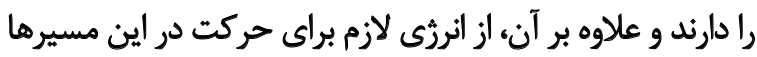

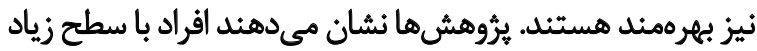

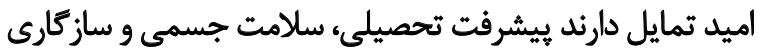

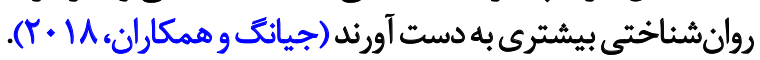

اميد همجنين با آسيبهاى روانشناختى رابطه معكوس يبيدا

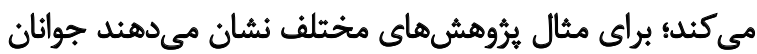

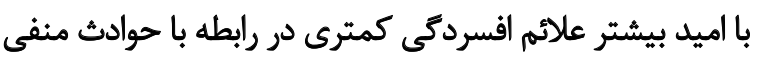

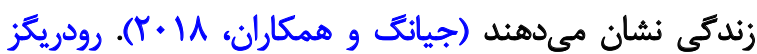

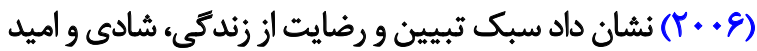

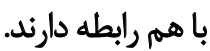

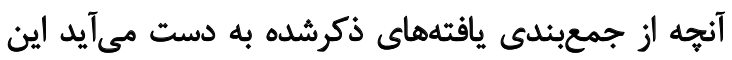

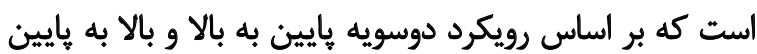

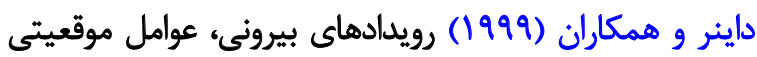

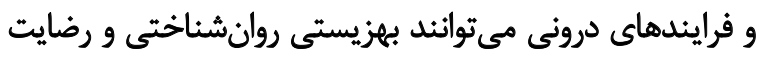

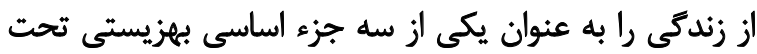

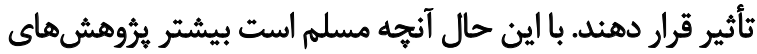

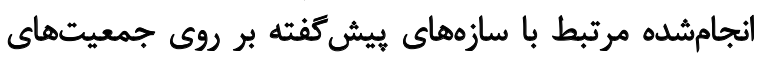

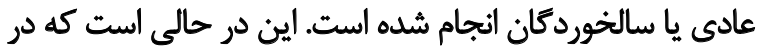

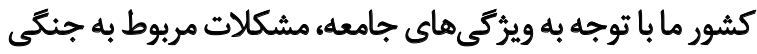

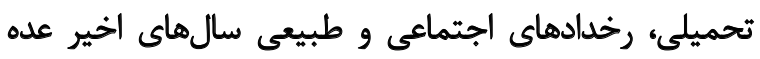

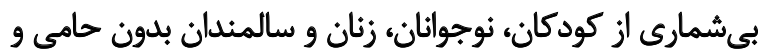

با سطوح سلامت روان به صورت مستقيم و غيرمستقيم ارتباط

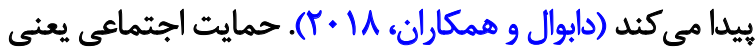

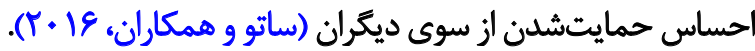

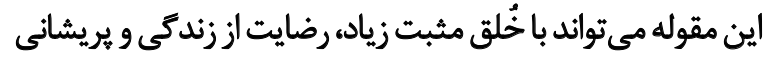

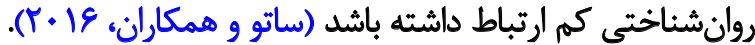

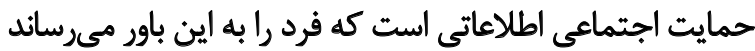

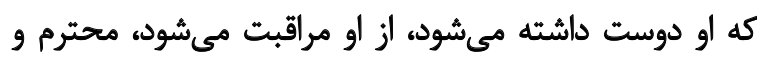

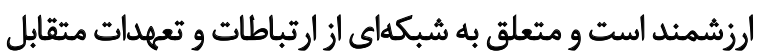

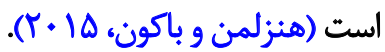

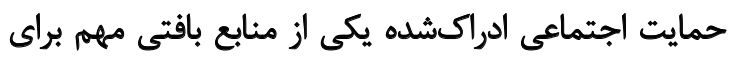

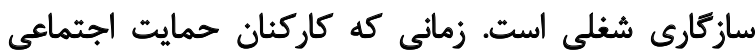

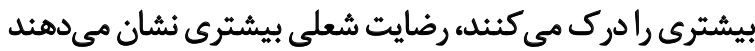

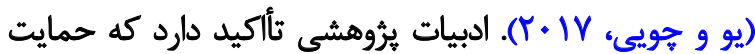

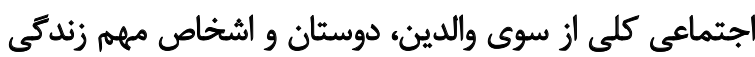

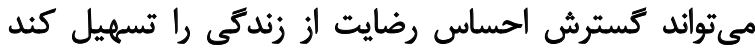

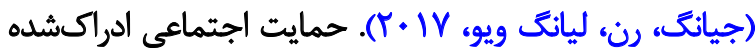

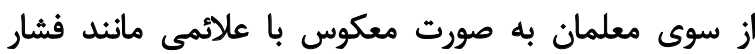

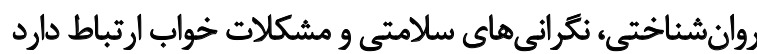

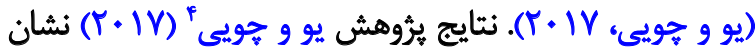

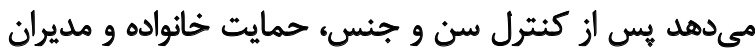

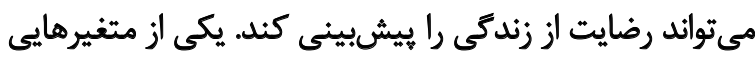

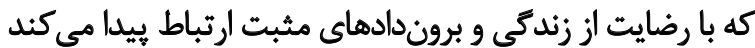

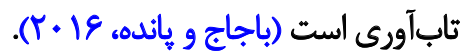

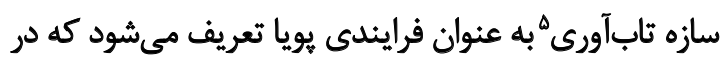

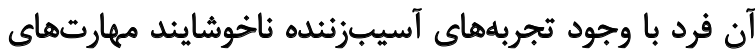

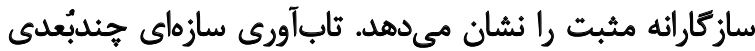

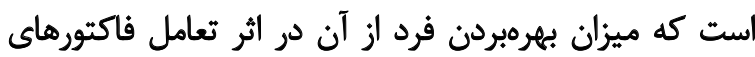

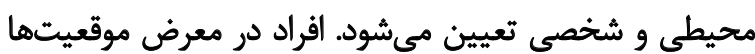

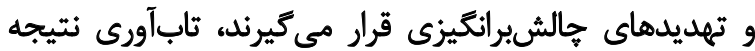

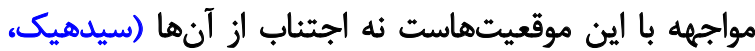

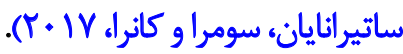

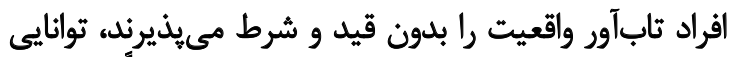

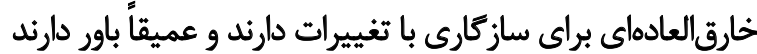

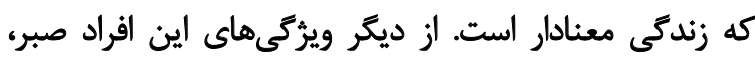

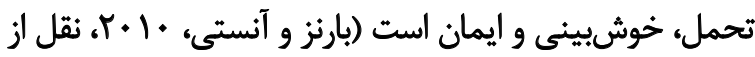

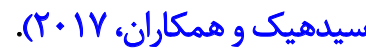

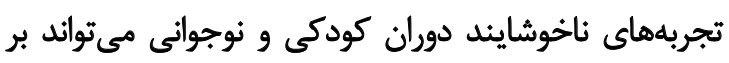

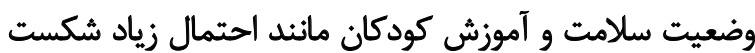

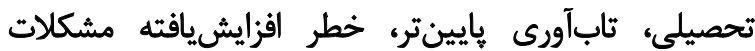
يادكيرى و رفتارى و افكار خودكشى تأثير داشتر افته باشد (سليمانيور، 


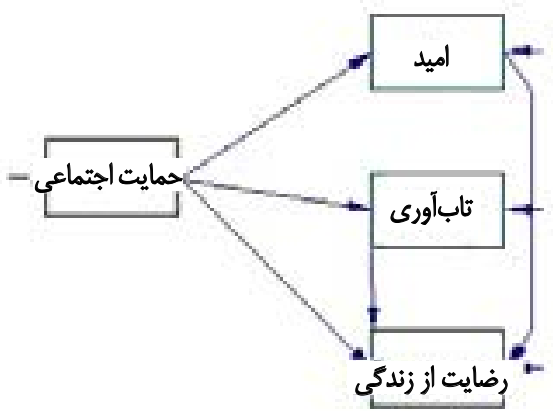

دوفصلنامه روانشناسى معاصر تهموير ا. مدل علّى رابطه حمايت اجتماعى ادراكشده با رضايت از زندكى با نقش واسطهاى ثابآورى و اميدوارى

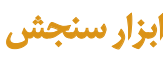

مقياس رضابت از زندكى

مقياس رضايت از زندگى براى اندازهيرى رضايت از زندكى

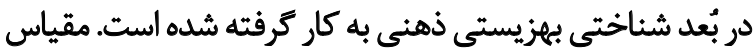

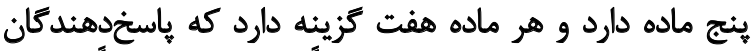

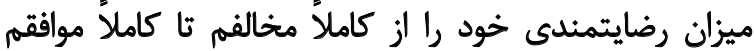

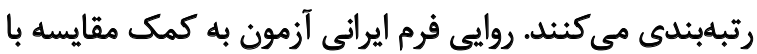

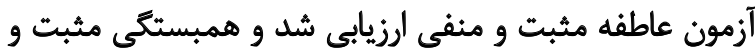

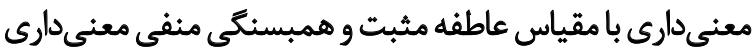

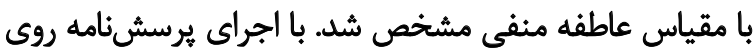

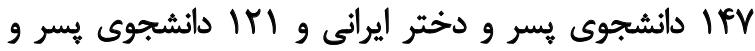

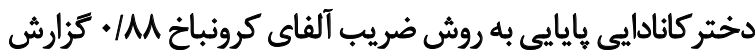

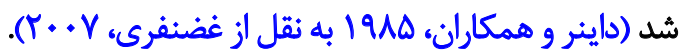

\section{مقياس جندبُعدى حمايتاجتماعى ادراكشده^}

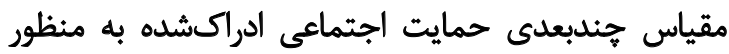

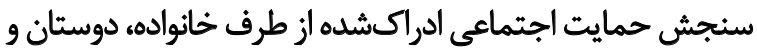

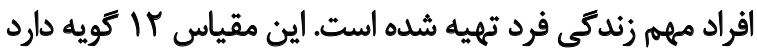

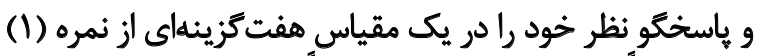

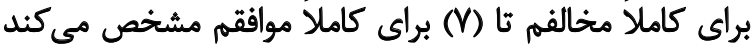

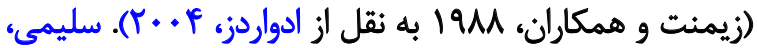

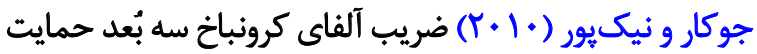

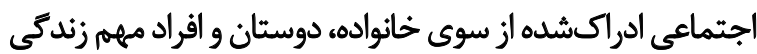

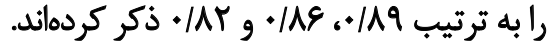

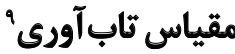

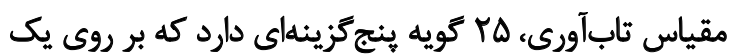

7. Satisfaction with Life Scale

8. Multidimensional Scale of Perceived Social Support

9. Resilience Scale
سريرست ماندهاند و در برابر بريشانيىهاى اجتماعى قادر به رفع

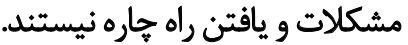
اكر افراد جامعه و به طور خاص قشر نوجوان جامعه دركير

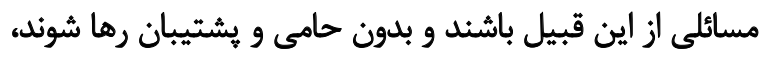

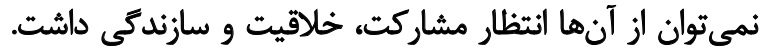

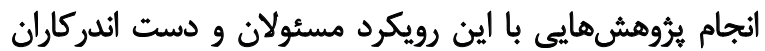

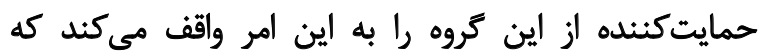

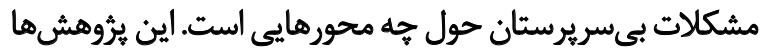

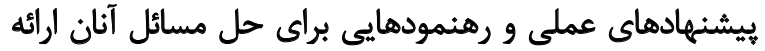

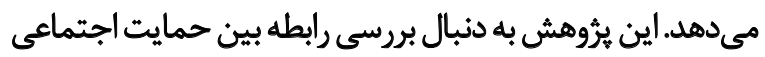

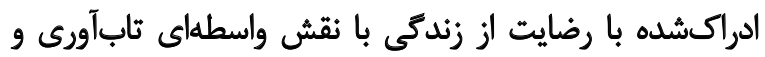
اميدوارى است (تصوير شماره ).

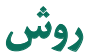

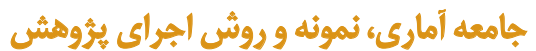

اين مطالعه از نوع همبستكى (معادلات ساختارى) است.

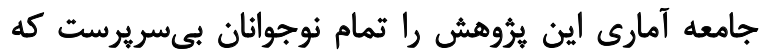

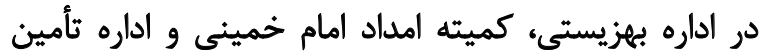

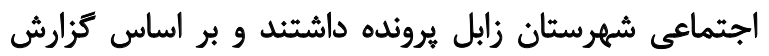

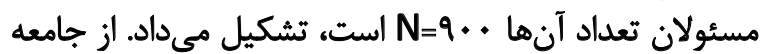

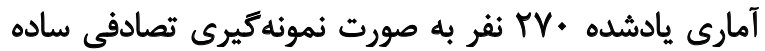

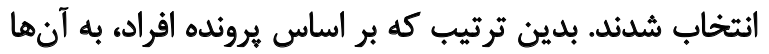

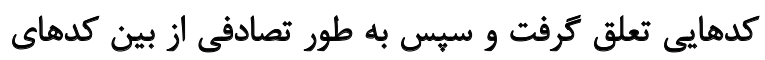

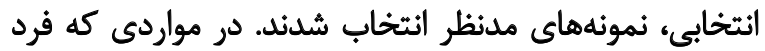

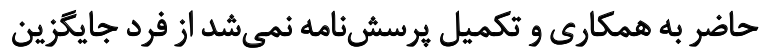

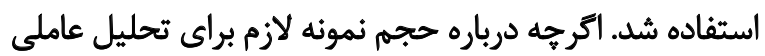

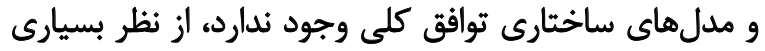

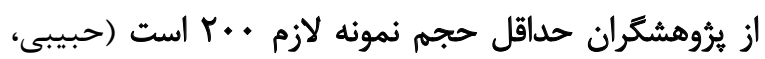

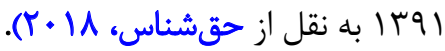


جدول ا. توزيع فراوانى متغير جنسيت

\begin{tabular}{|c|c|c|c|}
\hline درصد تراكمى & درصد & تعداد & جنسيث \\
\hline$\Delta Q \cdot F$ & $\Delta Q / \cdot F^{e}$ & IFV & يسر \\
\hline \multirow[t]{2}{*}{$1 .}$. & $p \cdot / q$ & $1 . r$ & لختر \\
\hline & $1 .$. & $r q q$ & كل \\
\hline
\end{tabular}

دوفصلنامه روايشناسى معاصر

جدول ז. ميانكين و ائحراف معيار متغيرهاى يُّوهش

\begin{tabular}{|c|c|c|c|}
\hline انحراف معيار & مياتكين & تعداد & هثغير \\
\hline NaF & TT/GS & \multirow{4}{*}{ req } & حمايت اجتماعى ادراكشده \\
\hline $91 .$. & $P F / M^{2}$ & & اميلوارى \\
\hline $\mid r / \pi$ & AV/IV & & ثاب آورى \\
\hline$F / A \Delta$ & $|E| A \mid$ & & رضايت از زندكى \\
\hline
\end{tabular}

دوفصلنامه وواثشناسى مeاصر

جدول r. بررسى مفروضه عادىبودن متغيرهاى يُروهش

\begin{tabular}{|c|c|c|}
\hline \multicolumn{2}{|c|}{ آزمون شاييرو ويلك براى عادىبودن } & \multirow{2}{*}{ متغيرها } \\
\hline سطح معنى دارى (P) & آماره & \\
\hline . M &.$/ 994$ & حمايت اجتماعى ادراكشده \\
\hline . /APT & ./99 & اميلووارى \\
\hline $.1 \cdot r \Delta$ & .198 & تاب!آورى \\
\hline . $A T I$ & ./994 & رضايت از زندكى \\
\hline rTr & . & حمايت اجتماعى ادراكشده \\
\hline
\end{tabular}

دوفصلنامه روانشناسى معاصر

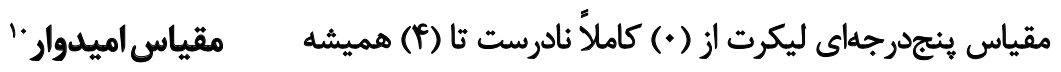

مقياس اميدوارى صفتى مقياسى كآيتمى است كه شامل

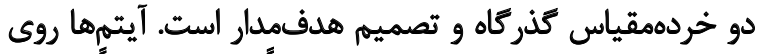

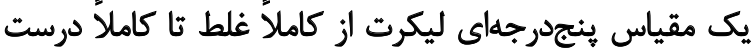

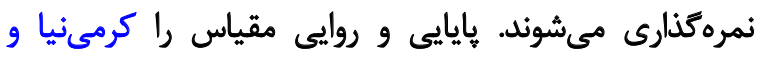

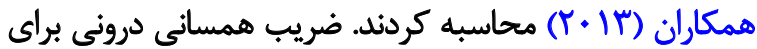

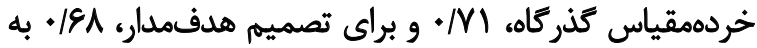

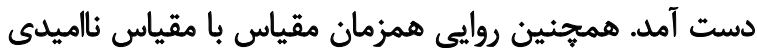

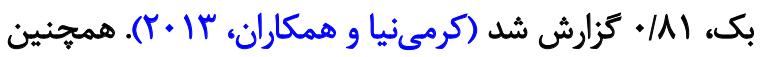

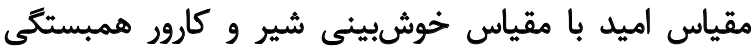

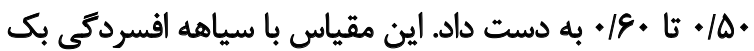

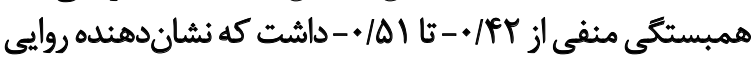

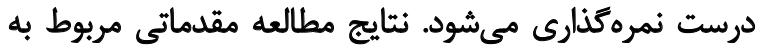

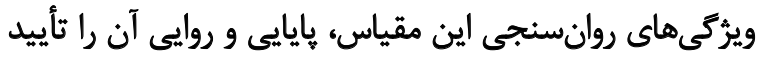

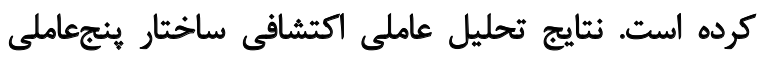

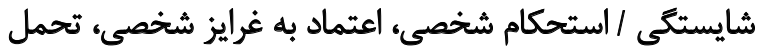

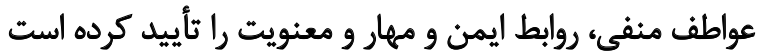

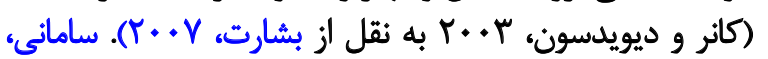

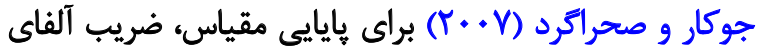

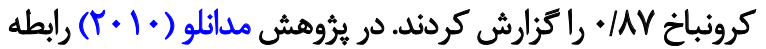

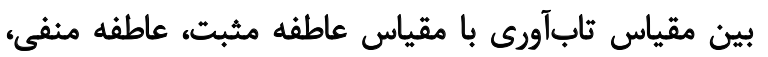

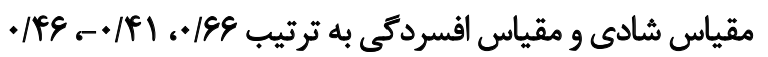
و و\&\&/-- به دست آمد كه نشاندهنده روايى مقياس است. ملاكى خوب اين يرسشنامه است (بيجارى و همكاران، و +. (Y). 
جدول †. ماتريس همبستكى متغيرهاي ثيروهش

\begin{tabular}{|c|c|c|c|c|}
\hline رضايت از زندكى & تابآورى & الميدوالى & ادراى حمايت اجتماعى & مثنغير \\
\hline & & & 1 & ادراك حمايت اجتماعى \\
\hline & & 1 &.$|p|$ & اميدوارى \\
\hline & 1 &.$/ 19$ &.$/ 4 q$ & تابآورى \\
\hline 1 &.$/ \pi \Delta$ & $\cdot / N_{1}$ &.$/ r$ & رضايت ازز زندكي \\
\hline
\end{tabular}

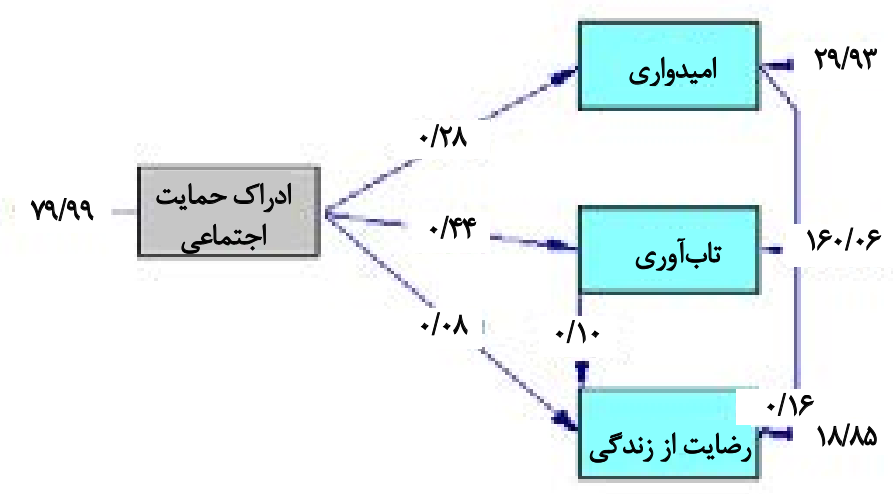

Chi-Square $=1.36, \mathrm{df}=1, \mathrm{P}=0.24373, \mathrm{RMSEA}=0.038$

دوفصلنامه ورانشناسى معاصر

تصوير r. ضرايب مسير مدل فرضى در مدليابى رضايت ازز زندكى

بررسى شد. از مهمثرين اين مفروضهها مفروضه عادىبودن Latolen

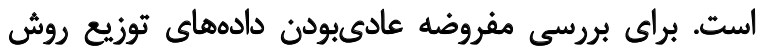

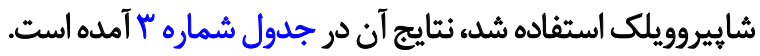

جدول شماره f نشان مى ندهد همبستكى حمايت اجتماعى

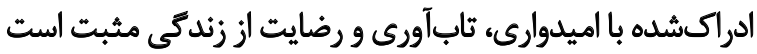

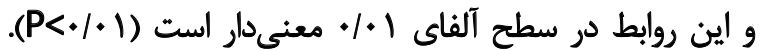

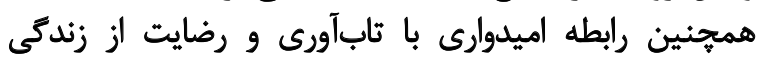

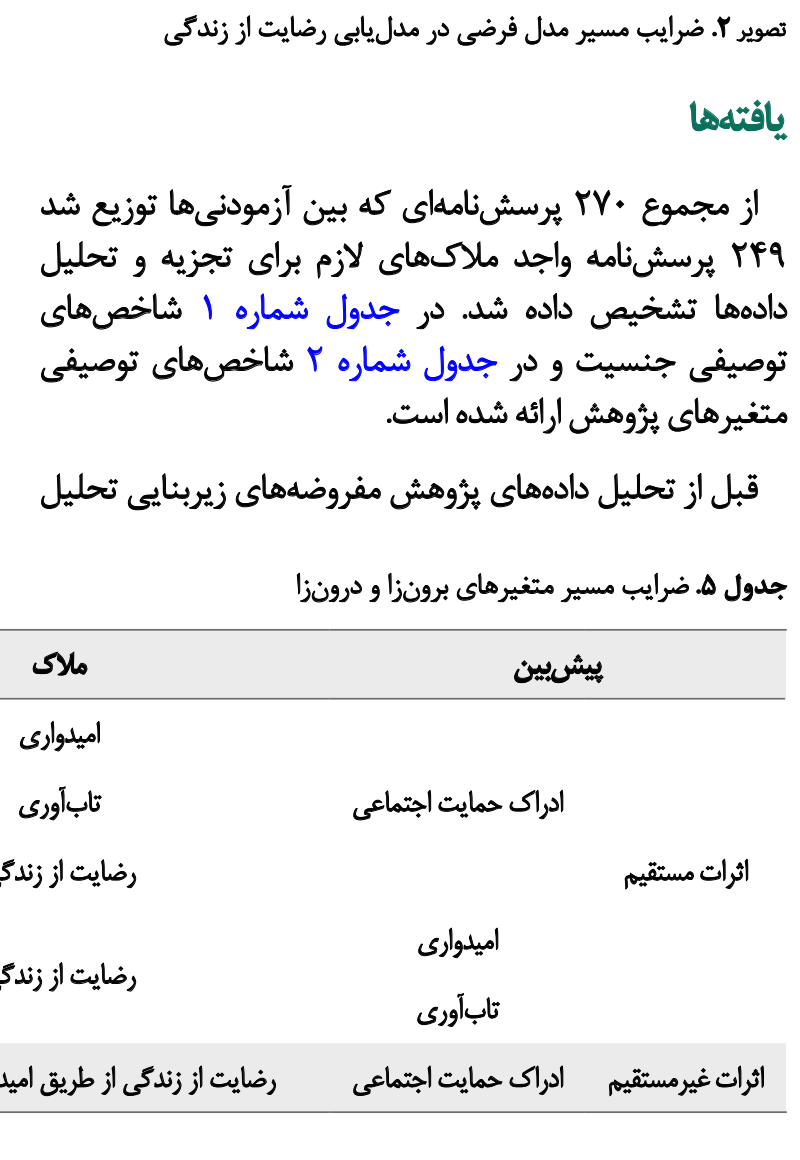


جدول و. شاخصهاي برازندگى مدل مفهومى يُروهش

\begin{tabular}{|c|c|c|c|c|}
\hline نتيجه & ميزان در مدل & جه زمانى مدل برازنده است؟ & 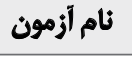 & رديف \\
\hline 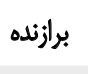 & V/rq & معنادار نباشد & $\chi^{\gamma}$ & 1 \\
\hline 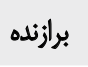 & $\%$ & اكر كوجكتر از هـ/. باشد. & RMSEA & r \\
\hline برازئده & $V / \cdot$. & بايد بزركتر از هa/. باشد. & GFI & r \\
\hline برازئده & $v / \cdot \cdot$ & بايد بزركتر از هو/. باشد. & $\mathrm{CFI}$ & r \\
\hline برازئده & .199 & بايد بزركتر از هو/. باشد. & NFI & $\Delta$ \\
\hline 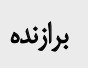 & $1 / \cdot \cdot$ & بايد بزركتر از هو/. باشد. & IFI & 8 \\
\hline 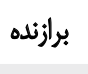 & .194 & بايد بزركتر از هو/. باشد. & NNFI & $\checkmark$ \\
\hline برازئده &.$/ 9 Y$ & بايد بزركتر از هو/. باشد. & AGFI & $\wedge$ \\
\hline 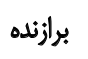 &.$/ 98$ & بايد بزركتر از هو/. باشد. & RFI & 9 \\
\hline
\end{tabular}

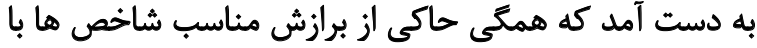

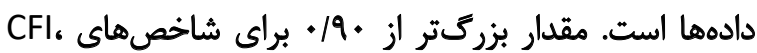
GFI، AGFI

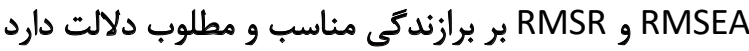

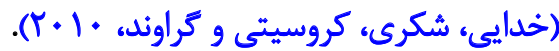

بـ

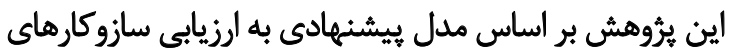

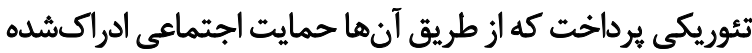

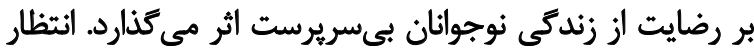

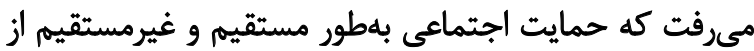

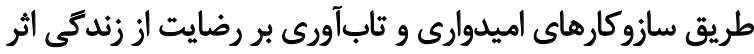

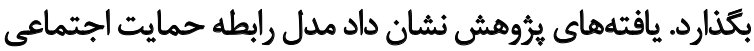

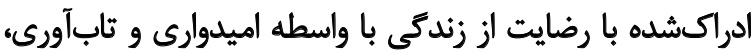

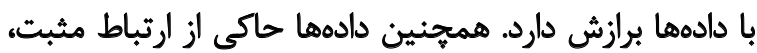

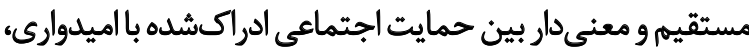

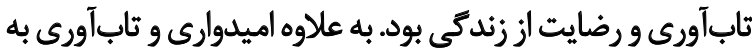

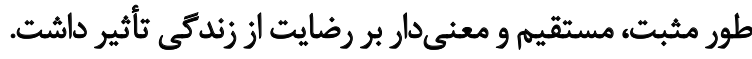

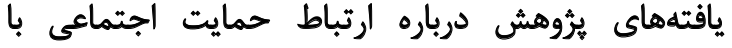

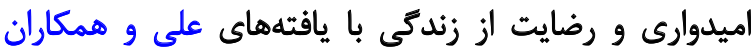

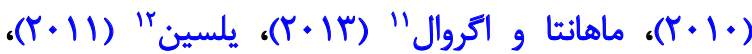

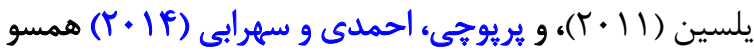

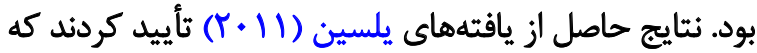

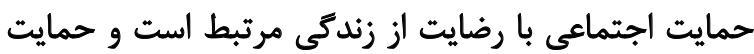

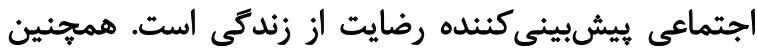

11. Mahanta, Aggarwal

12. Yalcin

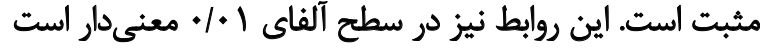

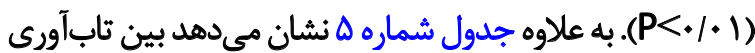

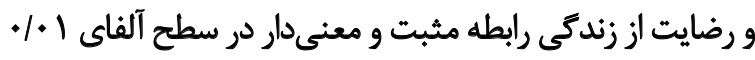

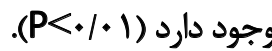

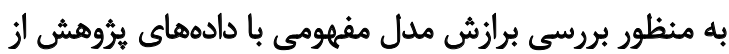

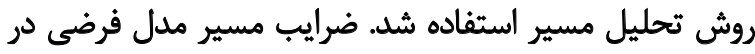

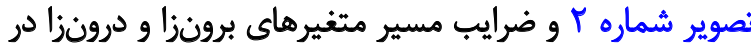

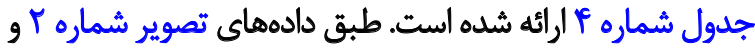

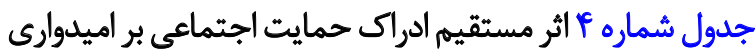

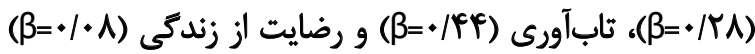

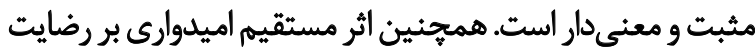

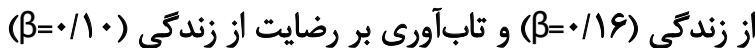

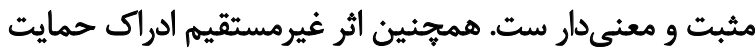

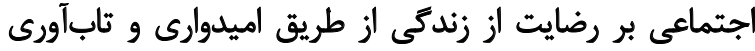

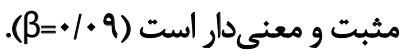

\section{تعيين بوازش الثقو}

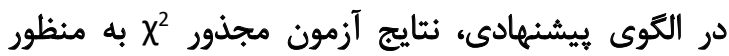

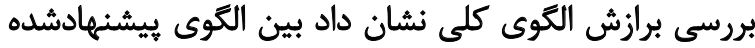

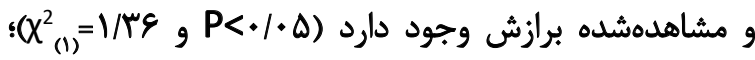

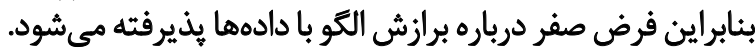

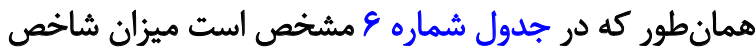

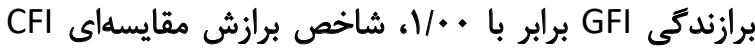

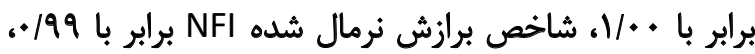

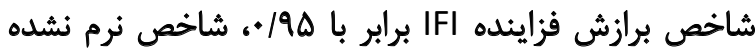

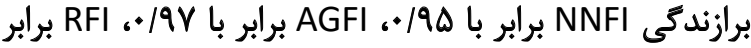

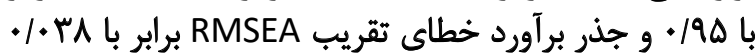




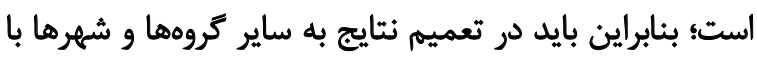

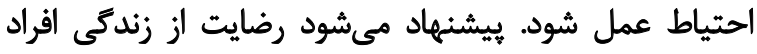

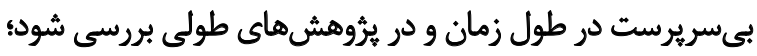

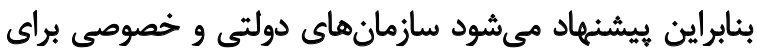

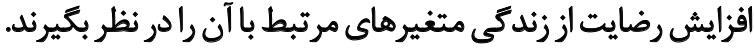

\section{ملاحظات الخلاقى}

\section{بيروى از اصول الخالق بيزوهش}

براى رعايت اصول اخلاقى همه شركت كنيندكان از اهداف

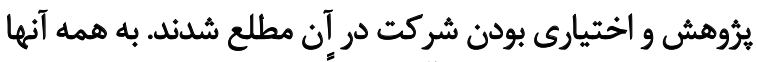

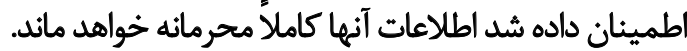

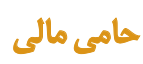

اين مقاله بركرفته از هاياننامه كارشناسى ارشد نويسنده سوم

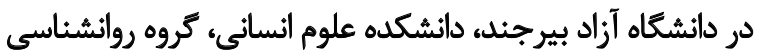

\section{مشاركت نويسند مكان}

تمامى نويسندكان در انجام اين بثروهش مشاركت داشتهاند.

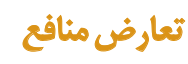

در اين يُروهش هيجّونه تعارض منافعى وجود ندارد.

$$
\text { كباسيكّز الوى }
$$

از تمامى نوجوانانى كه در اين يُوهش شركت كردند،

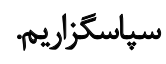

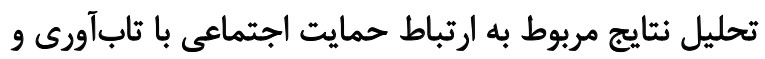

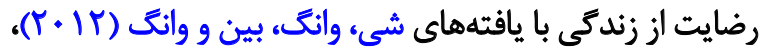

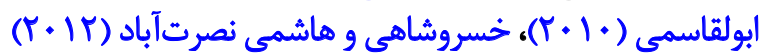

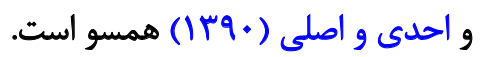

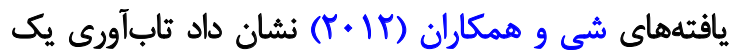

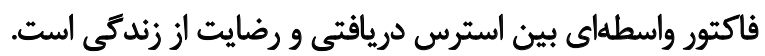

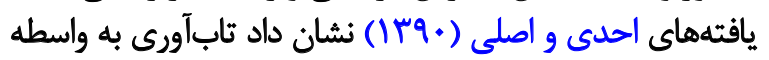
كاهش مشكلات هيجانى موجب افزايش كيفيت زندكى مى نشود.

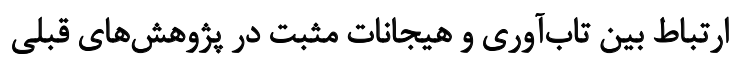

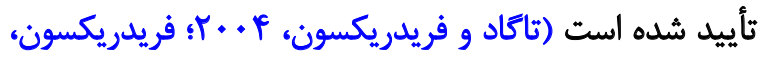

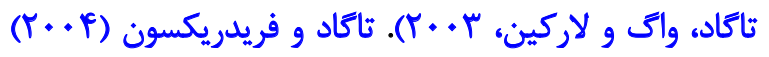

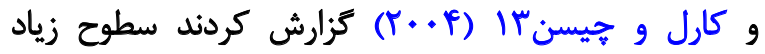

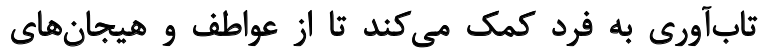

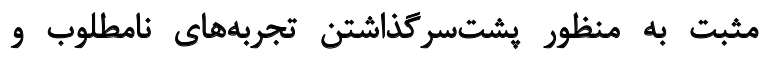

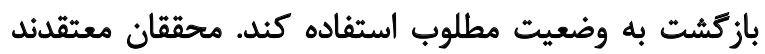

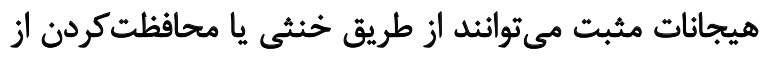

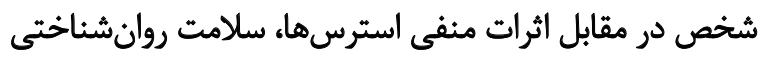
را ارتقا دهند (راتئ و همكاران، بات +r).

افراد با تابآورى بيشتر، منابع روانشناختى غنىترى ازئ جمله

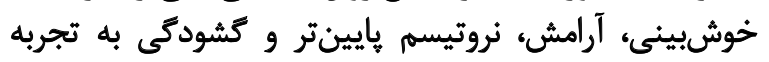

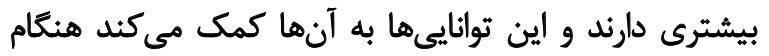

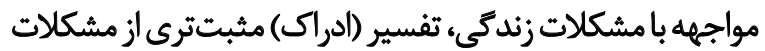

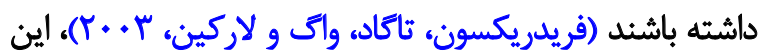

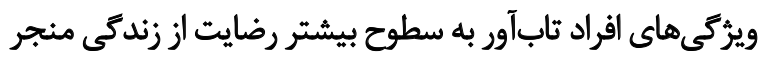

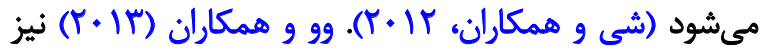

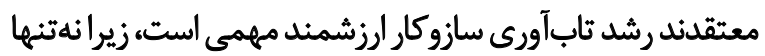

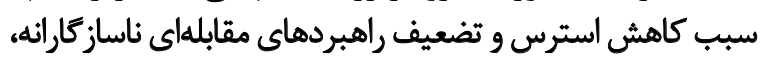

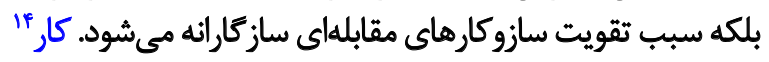

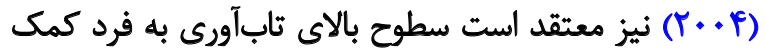

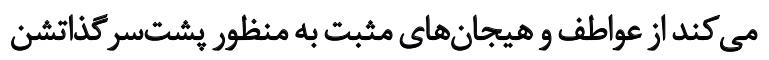

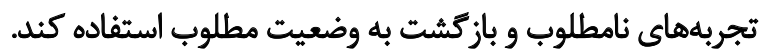

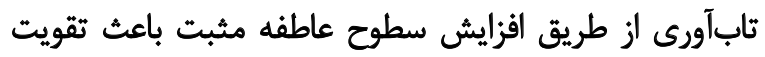

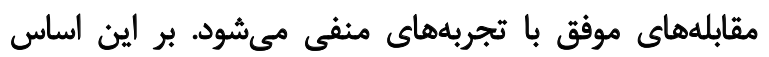

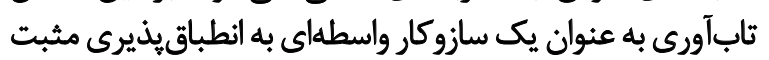

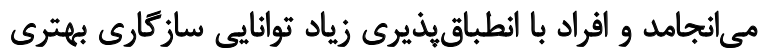

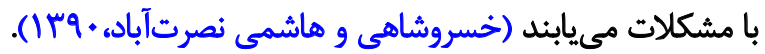

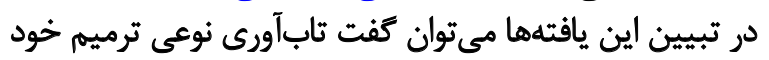

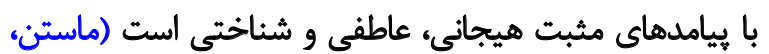

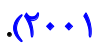
اين يُروهش روى نمونه محدودى و در شهر خاصى اجرا شده 


\section{References}

Abdolghasemi, A. (2011). [The relationship of resilience, self-efficacy and stress with life satisfaction in the students with high and low educational achievement (Persian)]. Quarterly Journal of Psychological Studies, 7(3), 131-52. [DOI: 10.22051/PSY.2011.1545]

Abolghasemi, A. (2010). Resilience and perceived stress: Predictors of life satisfaction in the students of success and failure. Procedia Social and Behavioral Sciences, 5, 748-52. [DOI:10.1016/j.sbspro.2010.07.178]

Ahadi, H., \& Asli, A. (2012). Prediction of quality of life of female students based on differentiation and resiliency variables (Persian)]. Woman and Family Studies, 4(14), 27-42.

Ali, A., Deuri, S. P., Deuri, S. K., Jahan, M., Singh, A. R., \& Verma, A. N. (2010). Perceived social support and life satisfaction in persons with somatization disorder. Industrial Psychiatry Journal, 19(2), 115-8. [DOI:10.4103/0972-6748.90342] [PMID] [PMCID]

Amani, S., Jokar, B., \& Sahragard, N. (2007). [Effects of resilience on mental health and life satisfaction (Persian)]. Iranian Journal of Psychiatry and Clinical Psychology, 13(3), 290-5.

Bajaj, B., \& Pande, N. (2016). Mediating role of resilience in the impact of mindfulness on life satisfaction and affect as indices of subjective wellbeing. Personality and Individual Differences, 93, 63-7. [DOI:10.1016/j. paid.2015.09.005

Besharat, M. A. (2007). Resilience, vulnerability and mental health. Psychscience, 6(24), 373-83.

Bijari, H., Ghanbari Hashem Abadi, B. A., Aghamohammadian Sherbaf Fatemeh, H. R., \& Homayi Shandiz, F. (2009). [Effects of hope-based group therapy on the promotion of hope in women with breast cancer (Persian)]. Ferdowsi University of Mashhad, 10(1), 171-84.

Carle, A. C., \& Chassin, L. (2004). Resilience in a community sample of children of alcoholics: Its prevalence and relation internalising symptomatology and positive affect. Journal of Applied Developmental Psychology 25(5), 577-96. [DOI:10.1016/j.appdev.2004.08.005]

Carr, A. (2004). Positive psychology: The science of happiness and human strength. New York: Brunner-Routledge.

Chen, W., Niu, G. F., Zhang, D. J., Fan, C. Y., Tian, Y., \& Zhou, Z. K. (2016). Socioeconomic status and life satisfaction in Chinese adolescents: Analysis of self-esteem as a mediator and optimism as a moderator. Personality and Individual Differences, 95, 105-9. [DOI:10.1016/j.paid.2016.01.036]

Diener, E., Suh, E. M., Lucas, R. E., \& Smith, H. L. (1999). Subjective wellbeing: Three decades of progress. Psychological Bulletin, 125(2), 276302. [DOI:10.1037/0033-2909.125.2.276]

Dobewall, H., Hakulinen, C., Keltikangas-Järvinen, L., Pulkki-Råback, L., Seppälä, I., Lehtimäki, T., et al. (2018). Oxytocin receptor gene (OXTR) variant rs 1042778 moderates the influence of family environment on changes in perceived social support over time. Journal of Affective Disorders, 235, 480-8. [DOI:10.1016/j.jad.2018.04.008] [PMID]

Edwards, L. M. (2004). Measuring perceived social support in Mexican American youth: Psychometric properties of the multidimensional scale of perceived social support. Hispanic Journal of Behavioral Sciences, 26(2), 187-94

Fredrickson, B. L., Tugade, M. M., Waugh, C. E., \& Larkin, G. R. (2003). What good are positive emotions in crisis? A prospective study of resilience and emotions following the terrorist attacks on the United States on September $11^{\text {th }}, 2001$. Journal of Personality and Social Psychology, 84(2), 365-76. [DOI:10.1037/0022-3514.84.2.365] [PMID] [PMCID]
Ghazanfari, F. (2007). [Intercultural well-being from an intercultural perspective (Persian)]. Psychology, 4(10), 406-21.

Gloria, A. M., Castellanos, J., \& Orozco, V. (2005). Perceived educational barriers, cultural fit, coping responses, and psychological well-being of Latina undergraduates. Hispanic Journal of Behavioral Sciences, 27(2), 161-83. [DOI:10.1177/0739986305275097]

Haghshenas, L. (2018). [The mediating role of social support in predicting general health based on emotional control in mothers of children with cancer (Persian)]. Contemporary Psychology, 12(1), 85-98.

Heintzelman, S. J., \& Bacon, P. L. (2015). Relational self-construal moderates the effect of social support on life satisfaction. Personality and Individua Differences, 73, 72-7. [DOI:10.1016/j.paid.2014.09.021]

Inzlicht, M., Aronson, J., Good, C., \& McKay, L. (2006). A particular resiliency to threatening environments. Journal of Experimental Social Psychology, 42(3), 323-36. [DOI:10.1016/j.jesp.2005.05.005]

Jiang, Y., Ren, Y., Liang, Q., \& You, J. (2018). The moderating role of trait hope in the association between adolescent depressive symptoms and nonsuicidal self-injury. Personality and Individual Differences, 135, 137 42. [DOI:10.1016/j.paid.2018.07.010]

Jiang, Z., Wang, Z., Jing, X., Wallace, R., Jiang, X., \& Kim, D. S. (2017) Core self-evaluation: Linking career social support to life satisfaction. Personality and Individual Differences, 112, 128-35. [DOI:10.1016/j. paid.2017.02.070]

Karaminia, R., Ahmadi Tahour-Soltani, M., Bagherian-Sararoudi, R., \& Moulavi, z. (2013). [Psychometric properties of the trait hope scale in Iranian students (Persian)]. Journal of Reserch in Behavioral Sciences, 10(6), 406-21.

Khodaei, A., Shokri, O., Krositi, E., \& Gravand, F. (2010). [Factor structure and psychometric properties of the Identity Style Inventory (ISI) (Persian)]. Advances in Cognitive Science, 11(1), 40-8.

Khosro Shahi, J., \& Hashemi Nosrat Abad, T. (2012). Relation of hope and resilience with psychological well-being in students (Persian)]. Journal of Psychology Andishe va Rafter, 6(22), 41-50.

Kim, B., \& Jeong, J. (2017). Dynamics of adolescents' life satisfaction and effect of class rank percentile: Evidence from Korean panel data. Journal of Economic Psychology, 59, 8-28. [DOI:10.1016/j.joep.2017.01.002]

Kong, F., Wang, X., Hu, S., \& Liu, J. (2015). Neural correlates of psychological resilience and their relation to life satisfaction in a sample of healthy young adults. Neuroimage, 123, 165-72. [DOI:10.1016/j.neuroimage.2015.08.020] [PMID]

Liu, Y., Wang, Z. H., \& Li, Z. G. (2012). Affective mediators of the influence of neuroticism and resilience on life satisfaction. Personality and Individual Differences, 52(7), 833-8. [DOI:10.1016/j.paid.2012.01.017]

Mahanta, D., \& Aggarwal, M. (2013). Effect of perceived social support on life satisfaction of university students. European Academic Research 1(6), 1083-94.

Madanlu, F. (2010). [Investigating the relationship between resilience, coping strategies and social support with the psychological state of females (Persian)] [MA Thesis]. Ardebil: Mohaghegh Ardebili University.

Masten, A. S. (2001). Ordinary magic: Resilience processes in development. American psychologist, 56(3), 227-38. [DOI:10.1037/0003 066X.56.3.227] [PMID]

Parpuchi, B., Ahmadi, M., \& Sohrabi, F. (2014). The relationship between religiousness and social support with the satisfaction of university students' life (Persian)]. Journal Management System, 14(3), 60-8. 
Quevedo, R. J. M., \& Abella, M. C. (2011). Well-being and personality: Facet-level analyses. Personality and Individual Differences, 50(2), 206-11. [DOI:10.1016/j.paid.2010.09.030]

Rodriguze, D. (2006). Colombian happiness, hope on interdisciplinary look at the correlation between explanatory style, cultural and satisfaction. Journal of Personality and Social Psychology, 52(1), 1260-84.

Rutten, B. P., Hammels, C., Geschwind, N., Menne-Lothmann, C., Pishva, E., Schruers, K., et al. (2013). Resilience in mental health: linking psychological and neurobiological perspectives. Acta Psychiatrica Scandinavica, 128(1), 3-20. [DOI:10.1111/acps.12095] [PMCID]

Salimi, A., Jokar, B., \& Nikpour, R. (2010). [Resilience, mental health and life satisfaction (Persian)]. Iranian Journal of Psychiatry \& Clinical Psychology, 5(3), 81-102.

Samani, S., Jokar, B \& Sahragerd, N. (2007). Resilience, Mental Health, and Satisfaction with Life. Iranian Journal of Psychiatry and Clinical Psychology, 13(3), 290-5.

Sato, W., Kochiyama, T., Kubota, Y., Uono, S., Sawada, R., Yoshimura, S., et al. (2016). The association between perceived social support and amygdala structure. Neuropsychologia, 85, 237-44. [DOI:10.1016/j.neuropsychologia.2016.03.036] [PMID]

Shi, M., Wang, X., Bian, Y., Wang, L. (2015). The mediating role of resilience in the relationship between stress and life satisfaction among Chinese medical students: A cross-sectional study. BMC Medical Education, 15, 16. [DOI:10.1186/s12909-015-0297-2]

Sidheek, K. F., Satyanarayana, V. A., Sowmya, H. R., \& Chandra, P. S. (2017). Using the Kannada version of the connor davidson resilience scale to assess resilience and its relationship with psychological distress among adolescent girls in Bangalore, India. Asian Journal of Psychiatry, 30, 16972. [DOI:10.1016/j.ajp.2017.10.015] [PMID]
Soleimanpour, S., Geierstanger, S., \& Brindis, C. D. (2017). Adverse childhood experiences and resilience: Addressing the unique needs of adolescents. Academic Pediatrics, 17(7), S108-S14. [DOI:10.1016/j. acap.2017.01.008] [PMID]

Tugade, M. M., \& Fredrickson, B. L. (2004). Resilient individuals use positive emotions to bounce back from negative emotional experiences. Journal of Personality and Social Psychology, 86(2), 320-33. [DOI:10.1037/00223514.86.2.320] [PMID] [PMCID]

Wu, G., Feder, A., Cohen, H., Kim, J. J., Calderon, S., Charney, D. S., et al. (2013). Understanding resilience. Frontiers in Behavioral Neuroscience, 7, 10. [DOI:10.3389/fnbeh.2013.00010] [PMID] [PMCID]

Yalçın, İ. (2011). Social support and optimism as predictors of life satisfaction of college students. International Journal for the Advancement of Counselling, 33(2), 79-87. [DOI:10.1007/s10447-011-9113-9]

You, S., Lee, J., Lee, Y., \& Kim, E. (2018). Gratitude and life satisfaction in early adolescence: The mediating role of social support and emotional difficulties. Personality and Individual Differences, 130, 122-8. [DOI:10.1016/j.paid.2018.04.005]

Yuh, J., \& Choi, S. (2017). Sources of social support, job satisfaction, and quality of life among childcare teachers. The Social Science Journal, 54(4), 450-7. [DOI:10.1016/j.soscij.2017.08.002] 